\title{
So Many Meetings, So Little Time: Using IP Videoconferencing To Revitalize Statewide Committees
}

\author{
Nancy Lombardo \\ Systems Librarian \\ Spencer S. Eccles Health Sciences Library \\ University of Utah \\ Salt Lake City, Utah 84112-5890 \\ U.S.A \\ nancyl@lib.med.utah.edu
}

and

\author{
Carol Hansen \\ Professor \\ Stewart Library \\ Weber State University \\ Ogden, UT 84408-2901 \\ CHANSEN@weber.edu
}

\section{Introduction}

Internet technologies continue to transform the library profession and redefine how we communicate with professional colleagues and peers. IP (Internet Protocol) videoconferencing provides increased opportunities for librarians to engage in virtual teamwork and e-collaborations. The use of video conferencing by a consortium in Utah has revitalized committee work and continuing education within this large western U.S. state. Academic libraries in Utah are separated by hundreds of miles. With only thirteen higher education institutions in Utah, the Utah Academic Library Consortium (UALC) has been extremely effective in promoting valuable collaborations and cooperative resource sharing for decades. Many libraries have consistently benefited from important cooperative efforts. UALC librarians have worked together successfully in many areas including interlibrary loan, collection management, information literacy programming ${ }^{1}$, library services to distance learners, ${ }^{2}$ and chat reference. The collaborative library work environment in Utah requires librarians from St. George (not far from Las Vegas) to serve on committees with librarians from Logan (near Idaho). These cities are nearly 400 miles apart. Recent budget shrinkages have caused many libraries to cut or eliminate their travel budgets. This makes committee work particularly challenging and threatens to discourage participation in collaborative projects across the state. Imagine having the choice between driving five hours one way to attend a one-hour committee meeting and attending that same meeting in your own office. This is the choice UALC librarians are now able to make when attending UALC committee meetings, due to implementation of an IP videoconferencing network among UALC libraries. Through the use of IP videoconferencing UALC committee members can effectively meet with colleagues across the state without leaving 
their own library.

\section{Funding and Equipment}

To alleviate the time and cost of UALC committee work, the Spencer S. Eccles Health Sciences Library proposed and received funding from the Marriner S. Eccles Foundation to purchase 16 Polycom ViaVideo units for distribution to all Utah academic libraries and the Utah State Library. The Spencer S. Eccles Health Sciences Library also works in collaboration with the Utah Telehealth Network (UTN). Through another Marriner S. Eccles Foundation gift, the Eccles Library purchased a Polycom MG323 12 port IP videoconferencing card which expanded an existing Accord MGC-100 multipoint bridge to 24 port capacity, allowing for videoconferences with up to 24 participants. The cost of each Polycom ViaVideo unit was approximately $\$ 500$. Because Americans are less willing to travel by air in the post-September 11th environment, videoconferencing has become much more popular in many business and educational settings. ${ }^{3}$ There are many vendors of videoconferencing equipment: among them are Clearphone, Sony, Tandberg, and Polycom, supplier of the Utah equipment due to its high video quality rating. ${ }^{4}$ Online startups, such as eYak and Eyeball Network are also offering alternatives.

\section{History of Project}

The Polycom video units were sent to each academic library in Utah during the Fall of 2002. Initially, one or two people in each institution experimented with the units by communicating informally with the systems librarian at the Eccles Health Sciences Library. Subsequent to this successful trial period, UALC committees have been holding regular, virtual meetings as the Polycom units are available in all academic libraries. While the videoconferencing process required some education for users and the elimination of several minor technical barriers, UALC committee members are enthusiastically embracing this technology as an effective, efficient and fun way to continue long standing collaborations without the costs and hassles of travel. The first committee to use the system repeatedly was the UALC Professional Development Committee. The initial video meeting for this group was a joint committee meeting with the UALC Systems committee on November 7, 2002. Involving the Systems Committee from the start was a very successful strategy because this ensured systems personnel from each institution would be aware of the equipment and how it worked. The strategy also ensured that in-house technical support would be present when needed at each library.

The first meeting required a significant amount of start-up time, during which each new participant went through a predictable series of "Can your hear me?" questions followed by adjustment of camera and audio settings. Overall, participants encountered few difficulties, as the Polycom ViaVideo software and hardware (video camera) were easy to install and use. The design incorporates a multimedia processor and the camera into one unit, and the ViaVideo connects easily to a desktop or laptop PC through the USB connection, using a standard network connection. Although there were several recommendations for 
improvement after the meeting, the first UALC Professional Development committee meeting via video was deemed to be a success. Nine of eleven possible sites/libraries participated, saving many hours of driving time for each committee member who was able to participate fully without leaving his/her home institution. This committee has continued to meet via video on several additional dates.

\section{Meeting Process}

Hosting a successful video committee meeting requires careful planning of step-by-step procedures. Prior to any UALC meetings, each potential participant filled out a profile data sheet that was stored in the UTN office. These profiles include basic information such as name, contact information, and workstation IP address to be used for meeting connections. Each library is allowed to have multiple profiles. Some institutions have committee members "check-out" the video unit on the day of their meeting and connect it to their own workstation, making the meeting even more convenient and comfortable since participants can then "meet" in their own offices. Other institutions have located the video unit in a conference room that staff can schedule as needed. Prior to hosting a videoconference, the UALC committee chair contacts one of the videoconference coordinators at UTN or the Eccles Library and schedules a date when the bridge is available. The UALC committee chair then contacts committee members to request advance notice of who will be participating and which profiles will be in use. The participants are entered into a meeting "table" on the bridge in preparation for assembling virtually on the appointed day and time. On meeting day, each participant "calls" the bridge by entering the bridge IP into the Polycom interface. At most sites, the IP has been added to the address book, so a single click of the mouse makes the connection. The video software is voice activated, so the person who is speaking is the person who is seen by all.

During the course of initial meetings, participants discovered several technical and production issues. Some sites experienced problems connecting due to firewall obstructions at their location. Committee members worked with IT staff at these sites and were able to open the necessary ports for successful two-way sharing of both video and audio. (Ports required are: TCP 1720, TCP 3230-3231, and UDP 3230-3235.) Production issues involve adequate lighting and etiquette protocol. Committee chairs conduct a role call at the start of each meeting so all participants know who is in attendance. Because the units are voice activated, committee members are asked to state their name and location, which gives all participants a chance to see them in the display. As there is a very slight delay, committee members are asked to state their name and location each time they speak during the meeting. This gives the camera and bridge sufficient time to bring the speaker's picture to the screen so everyone sees the new speaker when he/she begins to comment about meeting issues.

The overarching UALC representative group, as well as UALC subcommittees such as the Professional Development Committee, Systems Committee, and Reference/Instruction Committees have now held several successful meetings using the videoconferencing equipment. The Professional Development Committee 
has found that members from within the Salt Lake City metropolitan area often prefer to gather at one location where they meet the more distant committee members through the Polycom. For the most distant sites, such as Dixie College Library in St. George, Utah (a five hour drive to Salt Lake City), Southern Utah University in Cedar City, Utah (a four hour drive to Salt Lake City) and Utah State University in Logan, Utah (a three hour drive to Salt Lake City) the technology has been indispensable in allowing them to fully participate in UALC committee work and collaborative projects without wasting hours on Interstate 15 . To further facilitate participation, a UALC Web site page (http://medstat.med.utah.edu /videoconference/ or http://www.ualc.net ) has been created with Polycom video instructions for committee chairs and participants. Although still a work in progress, the page provides committee chairs with enough information to plan a meeting and provides participants with guidelines about expected meeting structure, behaviors and etiquette. In addition, the webpage outlines recommended specifications for workstations intended to run the Polycom ViaVideo units. A list of committee profiles is available so participants can determine whether they wish to use an existing location at their institution, or submit a new profile for their preferred location.

\section{The Future}

The early success of this videoconferencing system ensures its continuing use by Utah librarians. As more of them have experience using the system they will gain greater comfort with using this new style of communication and format. As the cost of this equipment decreases, it is highly likely that within the next five years many Utah academic library managers involved in collaborative activities will have a video camera unit on their desktops. Not only has UALC used video systems to plan professional development events, the Professional Development Committee has presented these same events via live video Web broadcasts. Copies of these video broadcasts, available through Web delivered video archives, are proving useful to those unable to attend the actual event and for ongoing staff development and continuing education. Two recent presentations - on large project planning, and on copyright and the Technology, Education and Copyright Harmonization Act (TEACH Act)- are available for anyone to view from the UALC Web site (http://www.ualc.net. (The TEACH Act is recent U.S. legislation that has significant implications for U.S. educational institutions due to its redefinition of the use of copyright protected materials in distance education.)

\section{Conclusion}

This collaborative statewide project using IP videoconferencing has been extremely valuable and effective in promoting participation in UALC committee meetings and in revitalizing the continuing education committee. Committee members, who were previously unable to participate actively, due to constraints of time or distance, are now regular and vital participants. Members of all UALC committees have easily adapted to this new communication style and technology that expands relationships and reduces or eliminates the barriers of time and 
distance for academic librarians involved in statewide committee work. This IP videoconferencing project is a model for many types of collaborative work environments where participants are unable, or do not desire, to meet in a traditional face-to-face settings. Saving time, particularly driving time, greatly improves the productivity, efficiency and collegiality of academic librarians. As academic librarians it is our role to share new Internet technologies with our patrons. It makes perfect sense for us to use these technologies to expand our own professional relationships and enhance our collaborative work environment.

\section{Notes}

1. Hansen, C. and Lombardo, N.T. (1997). Toward the Virtual University: Collaborative Development of a Web-based course. Research Strategies, 15, 68-79.

2. Brunvand, A., Hansen, C., Kochan, C., Lee, D., McCloskey, K., Morrison, R. (2001). Consortium Solutions to Distance Education problems: Utah Academic Libraries Answer the Challenges. Journal of Library Administration 31, 75.

3. Alexander, S. (2001, September 24). Getting the Picture. Minneapolis Star Tribune, 10D.

4. Mr. Spacely is coming to town: The tech isn't perfect, but video conferencing's day is near. (2001). Fortune 142, 242.

\section{About the authors}

Nancy Lombardo is systems librarian at the Eccles Health Sciences Library and a coordinator for the UALC videoconferencing system. Carol Hansen is instruction services librarian at Stewart Library, Weber State University and chair of the UALC professional development committee, email: nancyl@lib.med.utah.edu 